\title{
Evolución de la historia clínica criminológica en el Ecuador
}

\section{Evolution of criminological clinical history in Ecuador}

María Fernanda Zambrano Pérez ${ }^{1}$, Edmundo Nicasio Chóez Chiliquinga², Mayra Viviana Barros Carvajal $^{3} \&$ Martha Magdalena Parra Parra ${ }^{4}$

\begin{abstract}
.
DOI: https://doi.org/10.33262/cienciadigital.v3i1.1.359

The Criminological Clinical History contains investigative data of a criminal act. At the international level, advances have been made in the criminological study, based on the use of criminological clinical history. Objective: To describe the evolution of the Criminological Clinical History in the Julio Endara Criminology Institute. Methodology: A retrospective, descriptive observational study of 400 histories, taken from the years between 1936 to 2016, was conducted. Results: Most changes occurred in the years 1959 and 1979. In 2005, the last update, only one was introduced. variable. Another finding was the high number of incomplete data, or nonexistent records. When comparing the variables proposed in this study, the greatest similarities were with the models of 1959 and 1979. Conclusions: A regression was observed in the update, since the greatest similarity found was between the proposal and the clinical history. criminology of the year of 1979.
\end{abstract}

Keywords: Clinical history, evolution, comparison, criminology

\section{Resumen.}

\footnotetext{
${ }^{1}$ Universidad Central del Ecuador. Correo-e: a15 fernanda@ hotmail.com

${ }^{2}$ Universidad Central del Ecuador. Correo-e: edmundo.choez@gmail.com

${ }^{3}$ Universidad Central del Ecuador. Correo-e: mvbcmedi7@ hotmail.com

${ }^{4}$ Universidad Católica de Cuenca. Correo-e: marthaparra20@hotmail.com
} 
La Historia Clínica Criminológica contiene datos investigativos de un acto delictivo. A nivel internacional han surgido avances en el estudio criminológico, basados en el uso de la historia clínica criminologíca. Objetivo: Describir la evolución de la Historia Clínica Criminológica, en el Instituto de Criminología Julio Endara. Metodología: Se realizó un estudio observacional descriptivo retrospectivo de 400 historias, tomadas de los años comprendidos entre 1936 al 2016. Resultados: La mayoría de cambios se dieron en los años 1959 y 1979. En el año 2005, última actualización, únicamente se introdujo una variable. Otro hallazgo fue el alto número de datos incompletos, o registros inexistentes. Al comparar las variables propuestas en este estudio, las mayores semejanzas fueron con los modelos de los años 1959 y 1979. Conclusiones: Se observó un retroceso, en la actualización, ya que la mayor semejanza encontrada, entre la propuesta, fue con la historia clínica criminológica del año de 1979.

Palabras claves: Historia clínica, evolución, comparación, criminología

\section{Introducción.}

La Criminología desde sus inicios se ha dedicado al estudio del delito, las causas y su reparación, en sintesis, se encarga del estudio del problema de la infracción, del infractor y de su tratamiento. El estudio de los problemas propiamente criminológicos, empezó por investigaciones en diversos dominios de la ciencia, sobre todo en la medicina, la estadística moral (judicial), la sociología y la psicología. Es en el decenio de los ochenta del siglo XX, cuando se fomenta el uso de la Historia Clínica Criminológica, como una herramienta de estudio, organizándola metodológicamente, a la manera de la clínica médica. En nuestro país los inicios de la Criminología surgieron al crearse el Instituto de Criminología en 1936, bajo dependencia del Ministerio de Gobierno Justicia y Cárceles, con la inmediata dirección del "Médico Director General de Prisiones", en estas condiciones se mantuvo el Instituto, hasta el 14 de Julio de 1944, en la que, mediante Decreto Supremo No. 401, se lo adscribe a la Facultad de Jurisprudencia de la Universidad Central del Ecuador. En 1959 se codifica la ley la cual ratifica su mantenimiento dentro de la Universidad Central del Ecuador donde continúa hasta la actualidad con el nombre de Intituto de Criminología Julio Endara, en memoria del Médico Psiquiatra pionero en esta ciencia en nuestro país.

\section{Fundamento teórico}

Aplicaciones, y funciones de la criminología

El diagnóstico clínico criminológico es un informe de carácter legal realizado mediante un proceso dinámico que permite, a quien realiza un análisis, establecer hipótesis sobre los factores explicativos de la conducta de un infractor. Esta hipótesis se va modificando 
conforme se van desvelando trastornos de personalidad, rasgos de comportamiento, características del delito, entorno cultural, familiar y socio-demográfico, nivel de planificación, y la relación víctima-victimario de forma interdisciplinaria.

La historia clínica criminológica

Incluye la descripción tanto de la versión del delito y la visualización que el supuesto victimario le da a la infracción cometida, además de toda una serie de elementos, mismos que permiten entender: ¿Cómo?, ¿Para qué?, ¿Por qué? y ¿de qué manera? se dio una conducta que ofendió a otra persona, lo que produce una alteracion en la dinámica personal y social del afectado, así como de la comunidad a la que pertenece. Por tal motivo, para ser un instrumento útil, requiere de claridad en su redacción, certeza en la aplicación de sus conceptos y honestidad en la toma de datos.

Proceso del análisis criminológico

La historia clínica criminológica analiza al sujeto en tres momentos distintos que abarcan: el momento antes de cometer la infracción, durante la comisión del hecho y el tercer momento incluye el pronóstico y evolución que se aprecia que tendrá el sujeto tras el diagnóstico y tratamiento, en síntesis es decir habla del pasado, presente y futuro de un sujeto. Dicho esto, el proceso de análisis clínico criminológico es un sistema de planificación de actividades, integrado por tres elementos: valoración, diagnóstico e implementación.

Manejo de la Criminología en Latinoamérica

A nivel de Latinoamérica, la Criminología refleja una combinación clínico-jurídica centrada en lo biológico, psicológico y sociológico y ha sido manejada en base a la realidad de cada país, realidades sicológicas, económicas e ideológicas, lo que ha provocado que esta tenga un manejo no cotidiano en algunos países, como es el caso del nuestro. En general la investigación criminológica que se practica en Latinoamérica, ha estado centrada principalmente al estudio de poblaciones penales condenadas, individuos que se encuentran recluidos, buscando en ellos las causas que los llevaron a cometer la infracción con la finalidad de encontrar la forma de reducir la criminalidad.

\section{Metodologia.}

El estudio es de un diseño observacional descriptivo y retrospectivo. Observacional porque se observó el comportamiento evolutivo, los cambios acaecidos durante el transcurrir del tiempo, en la Historia Clínica Criminológica; descriptivo por cuanto se realizó una recolección de datos en relación a las variables observadas dentro de la Historia Clínica Criminológica, y dichas variables de describieron para demostrar la situación en la que se encuentran actualmente comparada con sus inicios. Se estudiaron ejemplares de la Historia Clínica Criminológica desde un determinado periodo, comparadas con otras que se 
implementaron en el mismo Instituto y retrospectivo porque el estudio se realizó en referencia a un tiempo pasado, se realizó un análisis del modelo de Historias Clínicas Criminológicas manejadas el Instituto de Criminología Julio Endara de la Universidad Central del Ecuador, de un total de 11130 historias clínicas criminológicas hasta el año 2016, se decidió la toma de 5 ejemplares, al azar, por cada año, desde 1936, hasta el 2016 obteniendo un total de 400 ejemplares para el estudio. La información se obtuvo de forma directa, para su tabulación y análisis, se utilizó una estadística comparativa y descriptiva, en los datos generales de la muestra estudiada.

\section{Resultados}

En la tabla 1 se muestra las variables presentes en la primera Historia Clínica Criminológica creada en el Instituto en 1936, podemos observar que, ítems importantes cómo: Ingreso, anamnesis criminológica y tratamiento penitenciario, aún prevalecen en el modelo actual del 2005.

\begin{tabular}{lccc}
\hline \multicolumn{1}{c}{ Ítem } & $\begin{array}{c}\text { Aparición en } \\
\text { el modelo }\end{array}$ & $\begin{array}{c}\text { Desaparece } \\
\text { en el modelo }\end{array}$ & $\begin{array}{c}\text { Continúa en } \\
\text { el modelo }\end{array}$ \\
\hline Número de Historia & 1936 & & 2005 \\
Penado & 1936 & 1937 & \\
Nombres del Delincuente & 1937 & 1979 & 2005 \\
Ingresó el & 1936 & & \\
Salió el & 1936 & 1959 & \\
Huellas dactilares & 1936 & 2005 & \\
Huellas palmares & 1936 & 1979 & \\
Delito cometido & 1936 & 1959 & \\
Fotografías identificadoras & 1936 & 2005 & \\
Lugar donde cometió el delito & 1936 & 1959 & \\
$\begin{array}{l}\text { Antecedentes } \text { Individuales (historia del } \\
\text { delincuente) }\end{array}$ & 1936 & 1959 & \\
$\begin{array}{l}\text { Antecedentes familiares (constitución del } \\
\text { hogar ) }\end{array}$ & 1936 & 1959. &
\end{tabular}


Examen antropológico

Fotografías del examen antropológico

Examen fisiológico y médico

Examen psiquiátrico

Asistencia médica durante la reclusión

Examen psicológico (Kretschmer)

Anamnesis criminológica

Índice de peligrosidad (Estudio)

Tratamiento penitenciario
1936

1959

1936

1936

1936

1936

1936

1936

2005

1936

1936

1959

1959

1979

1959

1959

Tabla 1. Resumen de aparición, desaparición y continuidad de variables en la Historia Clínica Criminológica creada en 1936 en el Instituto de Criminología Julio Endara.

Fuente: Departamento de estadística del Instituto de Criminología Julio Endara, Historias Clínicas Criminológicas

Elaboración: Md. Ma. Fernanda Zambrano Pérez

En la tabla 2 se muestra las variables ingresadas en 1959, podemos observar que, ítems importantes cómo: lugar de la infracción e infracción, aún prevalecen en el modelo actual del 2005.

\begin{tabular}{lccc}
\hline Ítem & $\begin{array}{c}\text { Aparición } \\
\text { en el } \\
\text { modelo }\end{array}$ & $\begin{array}{c}\text { Desaparece en } \\
\text { el modelo }\end{array}$ & $\begin{array}{c}\text { Continúa en } \\
\text { el modelo }\end{array}$ \\
\hline Egreso & 1959 & 2005 & 2005 \\
Lugar de la infracción & 1959 & & \\
Informe del servicio social & 1959 & 1979 & \\
Herencia & 1959 & 2005 & \\
Somatometría & 1959 & 2005 & \\
Anamnesis Médica & 1959 & &
\end{tabular}


Reconocimiento para el tratamiento del 1959 1979 penado

Tabla 2. Resumen de aparición, desaparición y continuidad de variables en la Historia Clínica Criminológica modificada en 1959 en el Instituto de Criminología Julio Endara.

Fuente: Departamento de Estadística del Instituto de Criminología Julio Endara, Historias Clínicas Criminológicas

Elaboración: Md. Ma. Fernanda Zambrano Pérez

En la tabla 3 se muestra las variables ingresadas en 1979 y en el 2005, podemos observar que, el único ítem ingresado en el 2005 fue Historia Médica y Psiquiátrica.

\begin{tabular}{|c|c|c|c|}
\hline Ítem & $\begin{array}{l}\text { Aparición en } \\
\text { el modelo }\end{array}$ & $\begin{array}{l}\text { Desaparece } \\
\text { en el modelo }\end{array}$ & $\begin{array}{c}\text { Continúa } \\
\text { en el } \\
\text { modelo }\end{array}$ \\
\hline Datos Estadísticos & 1979 & 2005 & \\
\hline Nombres y Apellidos & 1979 & & 2005 \\
\hline Fecha de pérdida de la libertad & 1979 & & 2005 \\
\hline Resumen procesal & 1979 & & 2005 \\
\hline Fecha & 1979 & & 2005 \\
\hline Investigación Socio-Familiar & 1979 & 2005 & \\
\hline Examen Médico & 1979 & 2005 & \\
\hline Historia Psiquiátrica & 1979 & 2005 & \\
\hline Examen psicológico (Rorschach) & 1979 & & 2005 \\
\hline Examen psicológico (test estructurado) & 1979 & & 2005 \\
\hline $\begin{array}{l}\text { Estudio del estado psicológico del paciente el } \\
\text { momento de cometer el delito }\end{array}$ & 1979 & 2005 & \\
\hline Estudio del delito & 1979 & & 2005 \\
\hline
\end{tabular}


Tabla 3. Resumen de aparición, desaparición y continuidad de variables en las Historias Clínicas Criminológicas modificadas en 1979 y el 2005 en el Instituto de Criminología Julio Endara.

Fuente: Departamento de Estadística del Instituto de Criminología Julio Endara, Historias Clínicas Criminológicas

Elaboración: Md. Ma. Fernanda Zambrano Pérez

Otro hallazgo de interes durante este estudio, fue el alto numero de variables en las cuales la información estuvo incompleta, y en varias ocasiones sin ningun tipo de registro, pondremos como ejemplo el gráfico 1 que nos detalla en porcentajes el registro de datos completos en 100 Historias tomadas desde 1959 hasta 1978. Llama la atención el bajo porcentaje de registró en el examen psiquiátrico.

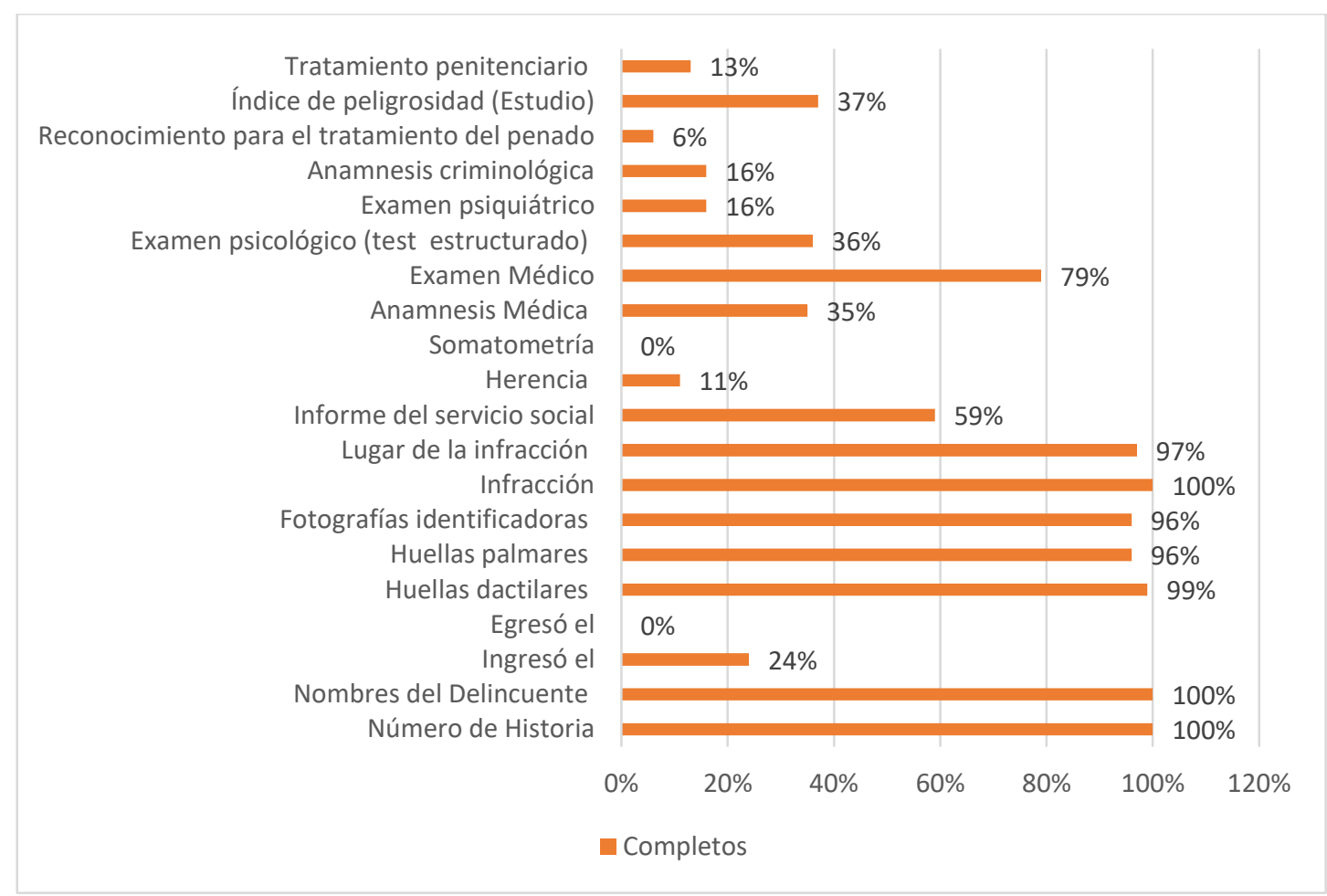

Gráfico 1. Variables con registro completo en la Historia Clínica Criminológica vigente desde 1959 hasta 1978, en el Instituto de Criminología Julio Endara

Fuente: Departamento de Estadística del Instituto de Criminología Julio Endara, Historias Clínicas Criminológicas

Elaboración: Md. Ma. Fernanda Zambrano Pérez 
La tabla 4 detalla las variables que son propuestas en este estudio para formar parte en un nuevo modelo, se puede ver claramente que las puntuaciones adquiridas son similares al comparar los modelos existentes desde 1936.

\begin{tabular}{llll}
$1936-$ & $1959-$ & $1979-$ & $2005-$ \\
1958 & 1978 & 2005 & 2016 \\
\hline
\end{tabular}

\begin{tabular}{|c|c|c|c|c|c|}
\hline \multirow{7}{*}{$\begin{array}{l}\text { Aspectos } \\
\text { generales }\end{array}$} & Número de Historia & $\checkmark$ & $\checkmark$ & $\checkmark$ & $\checkmark$ \\
\hline & $\begin{array}{l}\text { Fecha de apertura de la } \\
\text { Historia }\end{array}$ & & & & \\
\hline & $\begin{array}{l}\text { Fecha de actualización de la } \\
\text { Historia }\end{array}$ & & & & \\
\hline & Antecedentes Escolares & & & & \\
\hline & (Antes del ingreso a la & & & & \\
\hline & Cédula de Identidad & & & & \\
\hline & Huellas Dactilares & $\checkmark$ & $\checkmark$ & $\checkmark$ & \\
\hline \multirow{3}{*}{ Sociales } & $\begin{array}{lll}\text { Investigación } & \text { Socio }\end{array}$ & & & & \\
\hline & Familiar & & & $\checkmark$ & $\checkmark$ \\
\hline & Informe del servicio social. & & $\checkmark$ & & \\
\hline \multirow{6}{*}{$\begin{array}{l}\text { Criminológicos } \\
\text { (situación } \\
\text { Legal) }\end{array}$} & $\begin{array}{l}\text { Fecha de pérdida de la } \\
\text { libertad }\end{array}$ & & & $\checkmark$ & $\checkmark$ \\
\hline & Estudio del delito & $\checkmark$ & $\checkmark$ & $\checkmark$ & $\checkmark$ \\
\hline & Anamnesis criminológica & $\checkmark$ & $\checkmark$ & $\checkmark$ & $\checkmark$ \\
\hline & Victimología & & & & \\
\hline & $\begin{array}{l}\text { Estudio del estado } \\
\text { psicológico el momento de } \\
\text { cometer la infracción }\end{array}$ & & & $\checkmark$ & \\
\hline & $\begin{array}{l}\text { Índice de peligrosidad } \\
\text { (Estudio) }\end{array}$ & $\checkmark$ & $\checkmark$ & $\checkmark$ & $\checkmark$ \\
\hline \multirow{2}{*}{$\begin{array}{l}\text { Psicológicos y } \\
\text { Psiquiátricos }\end{array}$} & Evaluación psicológica & $\checkmark$ & $\checkmark$ & $\checkmark$ & $\checkmark$ \\
\hline & Evaluación psiquiátrica & $\checkmark$ & $\checkmark$ & $\checkmark$ & $\checkmark$ \\
\hline
\end{tabular}


Reconocimiento para el

tratamiento

\begin{tabular}{|c|c|c|c|c|c|}
\hline \multirow{4}{*}{$\begin{array}{c}\text { Médicos y } \\
\text { Odontológicos }\end{array}$} & Anamnesis médica & $\checkmark$ & $\checkmark$ & \multirow[t]{4}{*}{$\checkmark$} & \\
\hline & Anamnesis odontológica & & & & \\
\hline & /ficha odontológica & & & & \\
\hline & $\begin{array}{llr}\text { Asistencia } & \text { médica } & y \\
\text { odontológica } & \text { durante } & \text { la } \\
\text { reclusión } & & \end{array}$ & & & & \\
\hline & Puntuación & 8 & 9 & 11 & 8 \\
\hline
\end{tabular}

$\checkmark \quad$ : Se ha dado un valor de 1 para determinar una puntuación final.

Tabla 4. Semejanzas entre las variables propuestas por la investigadora y las variables existentes en los cuatro modelos analizados de Historia Clínica Criminológica desde 1936 hasta 2016 en el Instituto de Criminología Julio Endara Fuente: Departamento de Estadística del Instituto de Criminología Julio Endara, Historias Clínicas Criminológicas

Elaboración: Md. Ma. Fernanda Zambrano Pérez

\section{Conclusiones.}

- En nuestro país, la primera Historia Clínica Criminológica que se manejaba dentro del Instituto de Criminología Julio Endara, en su primera parte estaba destinada a datos que permitían la identificación del sujeto, fotografías de frente y de perfil, referencias generales e identificación dactiloscópica, en la segunda parte contenía la historia social del infractor, el estudio antropológico, integrando los estudios de Lombroso, en una tercera parte el estado mental y físico y una cuarta parte en la cual se hacía un seguimiento intrapenitenciario sobre el comportamiento y reeducación del individuo para su reinserción a la sociedad, procurando que aprendiera y desarrollara un oficio que le garantizaría un trabajo digno al recuperar su libertad teniendo los mismos objetivos a nivel internacional.

- Se debe mencionar además que, según estudios realizados en España tal cómo los mencionan, García, E y colaboradores, se recomienda que, los encargados de la elaboración de este documento deben ser: el correspondiente criminólogo, quien trabaja conjuntamente con otros peritos: psicólogos, pedagogos, sociólogos, médicos, odontólogos y otros que se requieran. En este punto se debe mencionar que en nuestro país al momento no se cuenta con suficientes profesionales en el manejo de esta ciencia y tampoco se cuenta con instituciones formativas en esta rama. En 1959, se realizaron algunos cambios en base, al avance de la Criminología, cambios como la 
sustitución del examen antropológico, remplazando algunas fórmulas métricas, nombrándolo: somatometría, y cambiando el término "delito" por el de "infracción", e inserción de un ítem importante; "reconocimiento del tratamiento del penado" que permitió identificar un adecuado tratamiento en caso de diagnóstico de alguna patología que haya sido la causa de la infracción. El siguiente cambio observado en el modelo de la Historia Clínica Criminológica fue en 1979, la mayoría de estos se dieron en base a los Derechos Humanos (1948), retirando el término "Nombres del Delincuente" por "Nombres y Apellidos", además de observarse cambios cómo: resumen procesal, estudio del delito e investigación socio-familiar, lo cual fue un importante avance en la estructuración de la Historia Clínica Criminológica ya que fue a la par con el avance observado a nivel latinoamericano, teniendo como ejemplo a países como Venezuela y Chile que registraron un cambio en los años 70. Es en el 2005, año en el que se registró la última actualización, desaparecen algunas variables cómo: el egreso de un centro penitenciario; huellas dactilares; datos estadísticos, además en este año se unifica la Historia psiquiátrica y médica, siendo este último el único nuevo aporte observado. Además, se ha visto una ausencia de ítems nuevos, con los que no se ha contado anteriormente y son importantes para el estudio criminológico. En la actualidad, a nivel de Latinoamérica existen países, como el nuestro, en los que ha existido un pobre avance de la Criminología, mismos que proponen como limitantes y problemas para el avance de esta ciencia, la falta de recursos, interferencias políticas y debilidades metodológicas, mientras que otros, como Colombia, han ampliado su visión dentro de este campo con grandes posibilidades y perspectivas, al empezar su uso, en base a estudios realizados por el FBI, para perfilaciones criminales en casos donde no exista un autor de la infracción.

- En conclusión deacuerdo con el objetivo tenemos que han sido cuatro los modelos de Historia Clínica Criminológica manejados por el Instituto de Criminología Julio Endara, vistos en los años 1936, 1959, 1979 y 2005; desde su creación, resalta el hecho qué, un gran número de datos estuvieron incompletos o inexistentes en algunas variables, fueron escasas las historias en las que se plasmó por completo la evolución y seguimiento del individuo; se puede observar en la comparación del modelo propuesto dentro de este trabajo con dos de los modelos utilizados en el instituto, que existe un retroceso ya que el modelo actual tiene menos semejanza que la observada en 1979; además se ha visto una ausencia de ítems nuevos, con los que no se ha contado anteriormente y son importantes para el estudio criminológico.

\section{Referencias bibliográficas.}

Arroyo SC. Principales instrumentos de medición de la peligrosidad criminal. Fundación UNIR. 2016 julio.

Cigarroa RV. Peligrosidad criminal: claves y conceptos para evaluarla. 2018. Available from: https://psicologiaymente.com/forense/peligrosidad-criminal. 
Falcone R. Dos casos paradigmáticos de la clínica criminológica de principios del siglo XX. V Congreso Internacional de Investigación y Práctica Profesional en Psicología XX. 2013.

García España E, Díez Ripollés J, Pérez Jiménez F, Benítez Jiménez MJ, Cerezo Domínguez AI. Evolución de la delincuencia en España: Análisis longitudinal con encuestas de victimización. Revista Española de Investigación Criminológica. 2010 Abril.

Gil DB. Criminología (II): evolución teórica: pasado, presente y futuro. Centro para el estudio y prevención de la delincuencia. 2016.

González E, Rodríguez R, Vera L. La aplicación de la criminología clínica en las investigaciones forenses actuales. Medicentro Electrónica. 2018 marzo; 22(1 ).

HIKAL W. El qué hacer de la criminología. Archivos de criminología, criminalística y seguridad privada. 2011.

Kirsch Ú. La construcción del criterio clínico criminológico. I Congreso Internacional de Investigación y Práctica Profesional en Psicología XVI. 2009. Available from: https://www.aacademica.org/000-020/103.pdf

León MA. Los dilemas de una sociedad cambiante: criminología, criminalidad y justicia en Chile contemporáneo (1911-1965). Revista Chilena de Historia del Derecho. 2012 2004;(19).

Louro Bernal dL, Serrano Patten. La investigación familiar y el valor de la metodología cualitativa para el estudio del afrontamiento a la enfermedad sicklemica. 2016. 1.

Miño DMG. Archivos de Criminología, Neuropsiquiatría y Disciplinas Conexas Quito: Universitaria; 2008.

Montes Prieto JL. Guía práctica para la elaboración del diagnóstico clínico. International EJournal of Criminal Sciences. 2012; 1(6 ).

Pont LMd. Criminología Latinoamericana. ILANUD. 1993. Available from: http://criminologiasur.blogspot.com/2011/12/origenes-de-la-criminologia-en.html

Rossi L, Navarlaz V. Transformaciones discursivas en el diseño de historias clinicasargentina. Revista de Historia de la Psicología en Argentina. 2013;(3 ).

Ruíz O, Robles García É. La expresión de la criminología clínica a través de la pericial criminológica. 1st ed. Andalucía: IC Editorial; 2017.

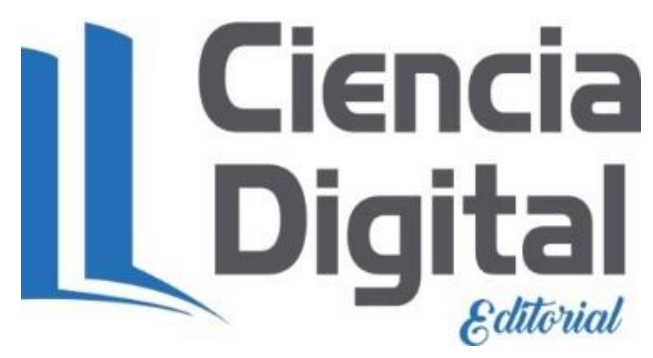




\section{Para citar el artículo indexado.}

Zambrano M., Chóez E., Barros M. \& Parra M. (2019) Evolución de la historia clínica criminológica en el Ecuador. Revista electrónica Ciencia Digital 3(1.1), 64-75. Recuperado desde: http://cienciadigital.org/revistacienciadigital2/index.php/CienciaDigital/article/view/359/777

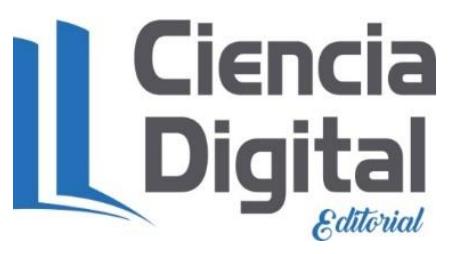

El artículo que se publica es de exclusiva responsabilidad de los autores y no necesariamente reflejan el pensamiento de la Revista Ciencia Digital.

El articulo queda en propiedad de la revista y, por tanto, su publicación parcial y/o total en otro medio tiene que ser autorizado por el director de la Revista Ciencia Digital.
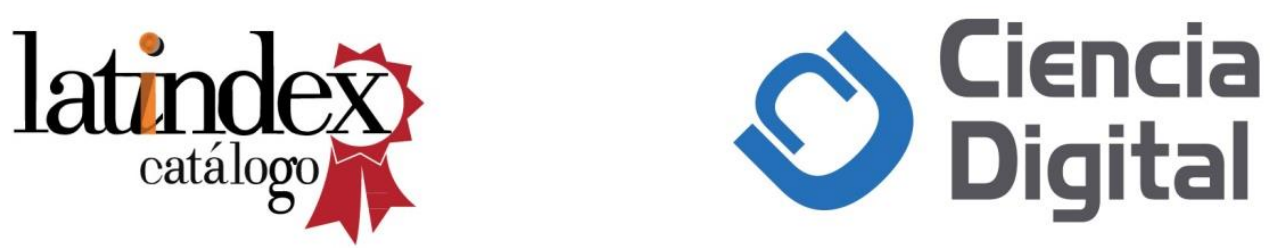